\title{
The possibility of difficult intubation in children with hydrocephalus undergoing shunt surgery
}

\author{
Ayten Saraçoğlu', Kemal Tolga Saraçoğlu', Ferhat Erenler', Hüseyin Canaz², \\ Deniz Kızılay', Ibrahim Alataş', i. Haluk Kafalı'
}

\author{
Department of Anesthesiology and Intensive Care', Neurosurgery ${ }^{2}$, Istanbul Bilim University \\ Medical School, Turkey
}

\begin{abstract}
Background and goals:
Hydrocephalus commonly cause serious challenges in airway management of newborn and pediatric patients, leading to enlarged head and distortion of skull anatomy. This result in difficulty for conventional laryngoscopy by hindering positioning of head. We aimed to determine the relationship between head circumference and the difficulties of intubation in pediatric patients undergoing shunt surgery.

Methods:

After obtaining the institutional board approval and written informed consent of parents, this study enrolled ASA class I-II, between the ages $0-18$ monhts 60 patients requiring ventriculoperitoneal shunt insertion. A standard monitorization, including oxygen saturation, electrocardiogram, noninvasive blood pressure, temperature measurement were implemented. Inhalational anesthesia induction was performed with $8 \%$ sevoflurane. Before the beginning of the surgical procedure; head circumference, the lower and upper lip-chin distance, sternomental and thyromental distance, neck extension, presence of macroglossia, Cormack-Lehane Classification scores were recorded. In addition, the number of attempts of successful intubation, the used endotracheal tube size, the presence of optimization manoeuvres, the presence of dental trauma were also recorded. The difficulty of intubation was evaluated with a $10 \mathrm{~cm}$ VAS ruler. All patients were intubated by the same anesthesiologist. The patients divided into three groups as and $>I$ month of age (group A, $n=20$ ), newborn (group $B, n=20$ ) and control (group $C, n=20$ ).
\end{abstract}

Table I: The comparison of demographics, physical examination, Cormack-Lehane Score, requirement for optimization maneuvre and a videolaryngoscope in newborns with and without hydrocephalus

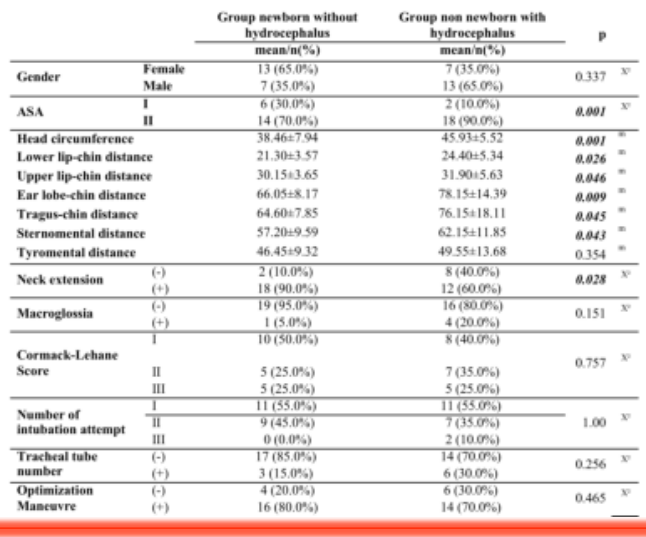

Results:

Head circumference, the lower and upper lip-chin distance, the ear lobe-chin distance, tragus-chin distance, and sternomental distance were significantly longer in group B (Table I). There was no statistically significant difference among groups regarding their demographics, Cormack-

Lehane scores, the presence of macroglossia and the requirement for optimization manoeuvres, the number of tracheal intubation attempts or a necessity of videolaryngoscope $(p>0,05)$. Compared with group $A$, head circumference, the lower and upper lip-chin distance, the ear lobe-chin distance, tragus-chin distance, and sternomental distance were significantly higher in group $B\left(p^{<} 0,05\right)$ and neck extension was also lower in group $B\left(p^{<} 0,05\right)$. There was no significant difference in number of intubation attempt between group $A$ and $B\left(p^{<} 0,05\right.$, Table 2$)$.

Table 2: The comparison of demographics, physical examination, Cormack-Lehane Score, requirement for optimization maneuvre and a videolaryngoscope in patients with hydrocephalus

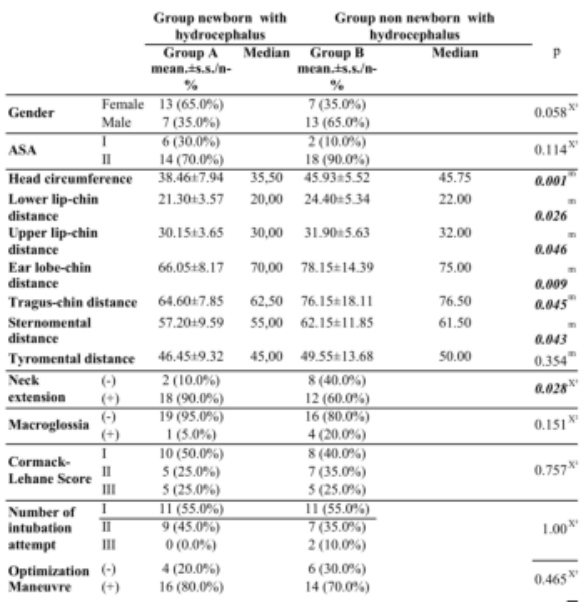

Conclusion:

Although the increased incidence of difficult intubation did not change in patients with hydrocephalus, the signs of difficult intubation were significantly higher in newborns.

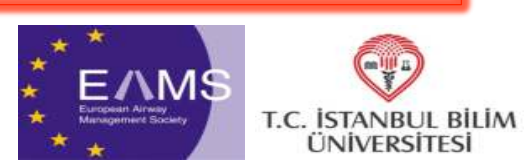

